EPiC Series in Computing
Volume 70, 2020, Pages 238-246
$\begin{gathered}\text { Proceedings of the 12th International Conference } \\ \text { on Bioinformatics and Computational Biology }\end{gathered}$

\title{
Predicting Microvascular Invasion of Hepatocellular Carcinoma by texture analysis of multi-phase MR image
}

\author{
Qin $\mathrm{Yu}^{1}$, Geng $\mathrm{Chen}^{3}$, Jiaqi $\mathrm{Li}^{4}$, Xiaolong $\mathrm{Liu}^{3}$, Xuegong Zhang ${ }^{4}$, Haiming$$
\mathrm{Lu}^{2 *}
$$ \\ ${ }^{1}$ BNRIST, Department of Automation, Tsinghua University, Beijing, China \\ ${ }^{2}$ BNRIST, Tsinghua University, Beijing, China \\ ${ }^{3}$ The United Innovation of Mengchao Hepatobiliary Technology Key Laboratory of Fujian \\ Province, Mengchao Hepatobiliary Hospital of Fujian Medical University, Fuzhou, China \\ ${ }^{4}$ Department of Automation, Bioinformatics Division, BNRIST and MOE Key Lab of \\ Bioinformatics, Tsinghua University, Beijing, China \\ yu-q18@mails.tsinghua.edu.cn, thestaroceanster@hotmail.com, \\ li-jq18@mails.tsinghua.edu.cn, xiaoloong.liu@gmail.com, \\ zhangxgetsinghua.edu.cn, luhmetsinghua.edu.cn
}

\begin{abstract}
Microvascular invasion (MVI) diagnosis is of vital importance in the curative treatment of hepatocellular carcinoma patients due to its close relationship with prognostic analysis. Currently, MVI detection often bases on surgical specimen, which is invasive for patients. This study, we extracted texture features of multi-phase MR image to predict the presence of MVI. Feature extraction employed neighboring gray level dependency emphasis (NGLDM) method, which is a common texture feature analysis method. Next, we built a SVM classifier to predict the presence of MVI using extracted features. Especially, multi-phase features were designed to enhance the precision of prediction. Enhanced MR images of pre-contrast phase and portal vein phase were used to extracting features. The method was tested by 5 -fold cross-validation on the dataset. The precision of prediction was $91.31 \%$, compared with the baseline method of $70.71 \%$. To make the prediction more interpretable, the relationship between NGLDM texture features and the presence of MVI was discussed in the end.
\end{abstract}

*Address correspondence to Haiming Lu (luhm@tsinghua.edu.cn) 
Predicting Microvascular Invasion of Hepatocellular Carcinoma by texture analysis of ... Q. Yu et al.

\section{Introduction}

Liver cancer is a big threat for human health. According to the data of global cancer statistic, liver cancer is ranked the seventh of new cancer cases. It is also the third cause of cancer death. The most common type is hepatocellular carcinoma (HCC), which comprising about $80 \%$ of the cases [1].

Several factors such as tumor size, presence of capsule affect the curative treatment for patients with HCC. Among these pathologic factors, microvascular invasion (MVI) is the most helpful one. It works in the evaluation of $\mathrm{HCC}$ grade before surgery as well as the prediction of postoperative recovery. Some published researches have shown that MVI has a close relationship with patients' survival period and the risk of recurrence after surgery $[2,3,4,5]$.

MVI diagnosis plays an important role in the curative treatment selection for patients of different conditions. However, traditional method of MVI diagnosis bases on the surgical specimen, which is invasive for patient. Also, the potential of preoperative evaluation is limited. Restricted sampling region cannot reflect the property of the whole tumor.

With the development of magnetic resonance image (MRI), some researchers turn to find radiomics paraments to predict MVI. Their studies use statistical method to show that tumor sharp and margin, tumor multifocality and DWI/T2 mismatch in MR image are associated to MVI prediction [6,7]. Though these methods are noninvasive, they seldom use the information from features of MVI itself. Also, these methods highly depended on the image analysis from experienced radiologists, which limits its research scale.

Deep learning is a new direction for MVI prediction. Deep features based on the Conventional Neutral Network (CNN) and Recurrent Neural Network (RNN) take advantage of combining multiphases information from MR image, which makes it get high accuracy in the prediction the presence of MVI and the diagnosis of malignant tumor $[8,9]$. But the black box pattern of deep learning makes it hard to do medical analysis based on the black-box features.

Texture features used to be common features for magnetic resonance image of brain tumor $[10,11]$. Recently, it has been wildly used to analysis the information hidden in magnetic resonance image of HCC. Texture features can be an effective predictor for the histological grading and early recurrence of HCC [12, 13]. It is also proved to be associated with the expression of some nuclear antigen [14]. Though those studies have shown the potential of texture features from MRI of HCC, they all focus on texture features of single phase separately.

The purpose of this article is to evaluate the possibility of using texture features to predict microvascular invasion of HCC. It also compares the performance of texture features from either single phase or multi-phases of MRI images and tried to find some explanation from the result.

\section{Materials and Method}

\subsection{Patients, Image and Data}

MR image data of this study came from Fujian Medical University. This retrospective study was approved by the local institutional review board and informed consents were signed by all patients. Thirty-one patients who were histopathological proved HCC were retrieved from September 2015 to November 2018 ( 28 males, 3 females, aged 56.96 \pm 10.68 , range from 30 to 75 years old). T1 enhanced MR images (Image size: 309 X 381, Voxel spacing: 1.2 X 1.2 X 3, from siemens MAGNETOM Verio) included pre-contrast phase, artery phase, portal vein phase and delay phase with masks of tumor position in each slice of four phases for every patient. Figure 1 shows a slice of tumor in pre-contrast phase and portal vein phase with its annotation. 

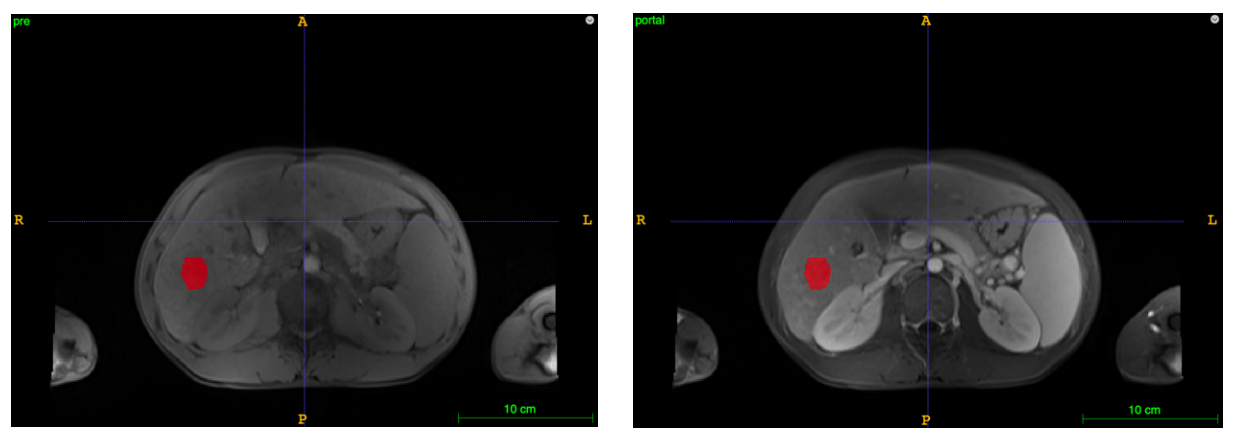

Figure 1: MR image in pre-contrast phase and portal vein phase with annotation

Of the thirty-one patients, twenty were diagnosed as MVI positive with the average of 17 effective slices of each patient. Eleven were MVI negative with the average of 13 effective slices. Taking each slice as an independent sample, there were 344 positive samples and 144 negative samples that could be split into training and test dataset according to five folds cross-validation method. Here a sample means an effective slice that has the annotation for tumor position.

\subsection{The Framework of the Method}

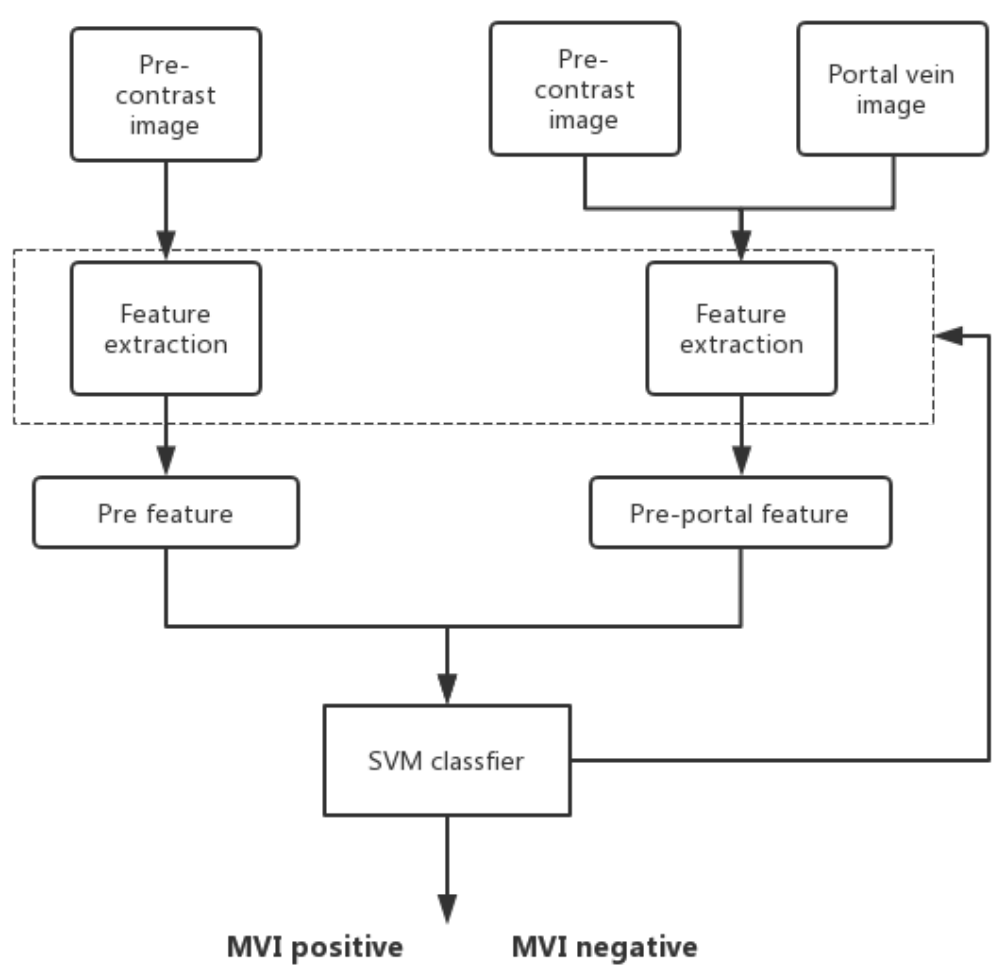

Figure 2: The flowchart of the method

The whole process of MVI classification method has been presented in Figure 2. 
Predicting Microvascular Invasion of Hepatocellular Carcinoma by texture analysis of ... Q. Yu et al.

As the flowchart shows, texture features for classification is composed of two parts: pre feature and pre-portal feature. Pre feature is the texture features extracted from pre-contrast phase of each sample. Pre-portal feature means the differential value between texture features of pre-contrast phase and portal vein phase. All these texture features were extracted in the tumor area (with annotation) and based on the neighboring gray level dependence matrix (NGLDM).

While training, the two parts of features need two steps to get best feature combination from dataset. The first step is selecting the best feature combination of pre feature independently, according to the MVI prediction result from classifier. Next, features are extracted from pre-contrast phase and portal vein phase and processed to get the differential value.

$$
\begin{gathered}
{\left[\begin{array}{ccl}
\text { Differential Value }_{1} & \cdots & \text { Differential value }_{n}
\end{array}\right]} \\
\quad=\left[\begin{array}{llll}
\text { Feature }_{1}^{\text {pre }} & \cdots & \text { Feature }_{n}^{\text {pre }}
\end{array}\right]-\left[\begin{array}{lll}
\text { Feature }_{1}^{\text {portal }} & \cdots & \text { Feature }_{n}^{\text {portal }}
\end{array}\right]
\end{gathered}
$$

This differential value would be combined with the best selection got in the first step and put into another adjustment based on the classifier accuracy. After finishing two steps, texture features which has combined the unique advantage of multi-phase of MRI could be used for MVI prediction. Finally, the combined texture features mentioned above would be imported into the SVM classifier to get the prediction result of MVI.

\subsection{NGLDM}

Neighboring gray level dependence matrix (NGLDM) is a common method of texture features analysis. It measures gray level dependence from calculating the elements in the neighborhood in the image matrix. NGLDM features are easily to computed while it can keep invariant under spatial rotation and linear gray level transformation [15].

NGLDM has various computed functions to measure gray level dependence from different aspects. Table 1 has listed a number of functions used in the feature extracted in this experiment [16].

Here:

$$
P(i, j) \in M_{N_{g} \times N_{d}}
$$

$P(i, j)$ is the gray level dependence matrix, $N_{g}$ is the number of gray level, $N_{d}$ is the number of dependence size.

In this task, microvascular invasion is a small-scale pathology phenomenon that is hardly identified by human eyes directly. But the integration of MVI could be presented in the image, especially in the

\begin{tabular}{|c|c|}
\hline $\begin{array}{l}\text { Function Name } \\
\text { Dependence Variance }\end{array}$ & Function Expression \\
\hline \multirow[t]{4}{*}{ Dependence Variance } & $\sum_{i=1}^{N_{g}} \sum_{j=1}^{N_{d}} P(i, j)(j-\mu)^{2}$ \\
\hline & $W=-\sum_{i=1}^{N_{g}} \sum_{j=1}^{N_{d}} P(i, j)$ \\
\hline & $\sum_{i=1}^{N_{g}} \sum_{j=1}^{N_{d}} P(i, j) j$ \\
\hline & $\overline{\sum_{i=1}^{N_{g}} \sum_{j=1}^{N_{d}} P(i, j)}$ \\
\hline Dependence Entropy & $D E=-\sum_{i=1}^{N_{g}} \sum_{j=1}^{N_{d}} \frac{P(i, j)}{\sum_{i=1}^{N^{\prime g}} \sum_{j=1}^{N_{d}} P(i, j)} \log _{2}\left(\frac{P(i, j)}{\sum_{i=1}^{N_{g}} \sum_{j=1}^{N_{d}} P(i, j)}+\epsilon\right)-$ \\
\hline
\end{tabular}
texture features. NGLDM features is able to reflect inner characteristics of microvascular invasion in the level of pixel, which could be useful to predict the presence of MVI. 
Predicting Microvascular Invasion of Hepatocellular Carcinoma by texture analysis of ... Q. Yu et al.

\begin{tabular}{lr}
\hline Function Name & Function Expression \\
\hline Small Dependence Emphasis & $S D E=\frac{\sum_{i=1}^{N_{g}} \sum_{j=1}^{N_{d}} \frac{P(i, j)}{j^{2}}}{\sum_{i=1}^{N_{g}} \sum_{j=1}^{N_{d}} P(i, j)}$ \\
Large Dependence Emphasis & $L D E=\frac{\sum_{i=1}^{N_{g}} \sum_{j=1}^{N_{d}} P(i, j) j^{2}}{\sum_{i=1}^{N_{g}} \sum_{j=1}^{N_{d}} P(i, j)}$ \\
Low Gray Level Emphasis & LGLE $=\frac{\sum_{i=1}^{N_{g}} \sum_{j=1}^{N_{d}} \frac{P(i, j)}{i^{2}}}{\sum_{i=1}^{N_{g}} \sum_{j=1}^{N_{d}} P(i, j)}$ \\
High Gray Level Emphasis & HGLE $=\frac{\sum_{i=1}^{N_{g}} \sum_{j=1}^{N_{d}} P(i, j) i^{2}}{\sum_{i=1}^{N_{g}} \sum_{j=1}^{N_{d}} P(i, j)}$ \\
Small Dependence Low Gray & $S D L G L E=\frac{\sum_{i=1}^{N_{g}} \sum_{j=1}^{N_{d}} \frac{P(i, j)}{i^{2} j^{2}}}{\sum_{i=1}^{N_{g}} \sum_{j=1}^{N_{d}} P(i, j)}$ \\
Sevel Emphasis & \\
Level Emall Dependence High Gray & $S D H G L E=\frac{\sum_{i=1}^{N_{g}} \sum_{j=1}^{N_{d}} \frac{P(i, j) i^{2}}{j^{2}}}{\sum_{i=1}^{N_{g}} \sum_{j=1}^{N_{d}} P(i, j)}$ \\
\hline
\end{tabular}

Table 1: Part of NGLDM functions

\subsection{Algorithm implementation}

The experiment was implemented in Python using several python packages including SimpleITK, PyRadiomics, Sklearn were employed. PyRadiomics is an opensource platform that provides radiomic feature extraction from medical image like CT, MRI and so on. ${ }^{[16]}$

At the beginning, MR images in DICOM format were transformed into NIFIT format (with suffix of .nii ) with ROI annotation. The original ROI annotation was labeled in pre-contrast phase and then copied into other phases. MR images of pre-contrast phase and portal vein phase were imported with their corresponding ROI annotation separately. After regular image preprocessing, the ROI images were well prepared to extract texture features. PyRadiomics could provide the value texture features according to the input task requirement. In this task, it was required to provide NGLDM features extraction, which is mentioned in Table 1, from single slice of MR images in pre-contrast phase or portal vein phase. Then these features were integrated for classification by Sklearn package and finally got the accuracy result.

\section{Experiment Result}

In order to evaluate the performance of the NGLDM features for MVI prediction of HCC patients, 5 folds cross-validation test was conducted based on 488 samples from 31 patients. The experiment result is demonstrated in Table 2. It consists of the average value of precision, recall and $\mathrm{fl}$ with respective standard deviation. 
Predicting Microvascular Invasion of Hepatocellular Carcinoma by texture analysis of ... Q. Yu et al.

\begin{tabular}{lccc}
\hline & Precision (\%) & Recall (\%) & F1 (\%) \\
\hline Tumor size & $70.71 \pm 6.25$ & $95.79 \pm 5.83$ & $81.17 \pm 4.93$ \\
Pre feature & $90.26 \pm 9.68$ & $96.06 \pm 2.81$ & $92.74 \pm 5.41$ \\
Pre feature \&Pre-portal feature & $91.31 \pm 8.72$ & $96.55 \pm 2.06$ & $93.59 \pm 4.60$ \\
\hline
\end{tabular}

Table 2: Performance of Tumor size, Pre feature and Pre \& Pre-portal feature

Here the result of Tumor Size means the experiment result that used tumor size as the only feature to predict the presence of MVI. In daily diagnosis, doctors often use tumor size as a prior evidence of MVI detection. Also, the positive relationship between tumor size and presence of MVI has been revealed. [17] So the result of Tumor Size is regarded as baseline to evaluate the promotion texture features brought to the prediction.

As the table shown, compared with the baseline method, Pre feature obviously improved the precision. It also increased the stability of recall. With the addition of Pre-portal feature, the precision was further enhanced, as well as its stability. The value of recall and fl were also slighted improved.

In each group of 5 folds, 5 MVI positive patients and 3 MVI negative patients were split into test data, and the remained 23 patients were used for training. Because the number of effective slices for each patient are different, number of samples could be various between different group. Detailed data are presented in Table 3 and Table 4.

\begin{tabular}{llrrrrr}
\hline & & Group 1 & Group 2 & Group 3 & Group 4 & Group 5 \\
\hline Training & Positive & 255 & 247 & 230 & 243 & 264 \\
samples & Negative & 97 & 104 & 119 & 93 & 102 \\
Test & Positive & 89 & 97 & 114 & 101 & 80 \\
samples & Negative & 47 & 40 & 25 & 51 & 42 \\
\hline
\end{tabular}

Table 3: Number of train and test data

\begin{tabular}{llrrrrr}
\hline & & Group 1 & Group 2 & Group 3 & Group 4 & Group 5 \\
\hline Pre feature & Precision & 98.88 & 72.31 & 91.38 & 90.09 & 98.67 \\
& Recall & 98.88 & 96.91 & 92.98 & 99.01 & 92.50 \\
& F1 & 98.88 & 82.82 & 92.17 & 94.34 & 95.48 \\
Pre feature & Precision & 98.88 & 75.59 & 91.45 & 90.65 & 100 \\
\& Pre-portal & Recall & 98.88 & 98.97 & 93.86 & 96.04 & 95 \\
feature & F1 & 98.88 & 85.71 & 92.64 & 93.21 & 97.44 \\
\hline
\end{tabular}

Table 4: Prediction performance in 5 folds cross-validation

The training process includes feature selection. Best feature combination for Pre feature is Small Dependence Emphasis, Dependence Nonuniformity Normalized, Small Dependence Low Gray Level Emphasis, Small Dependence High Gray Level Emphasis, Large Dependence Low Gray Level Emphasis. Based on the above feature, best combination for Pre-portal feature is Large Dependence Emphasis, Gray Level Variance and Dependence Entropy. The selection process is shown in Figure 3. 
Predicting Microvascular Invasion of Hepatocellular Carcinoma by texture analysis of ... Q. Yu et al.
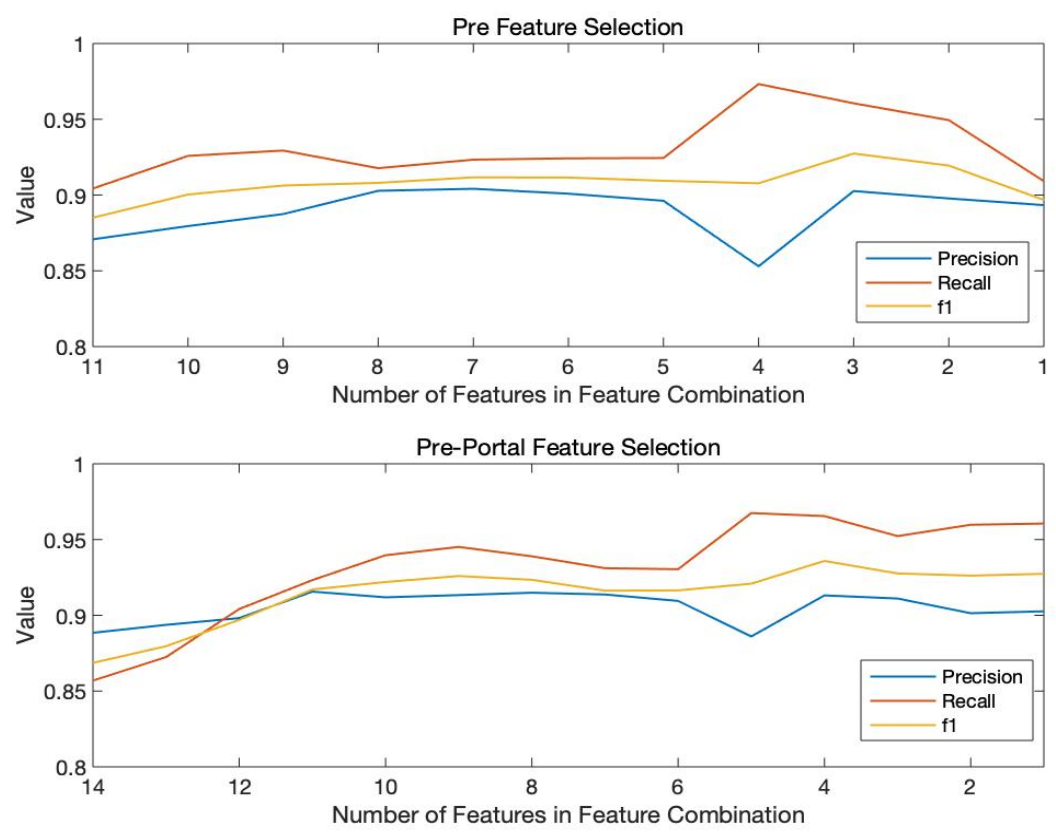

Figure 3: Indicator curves for feature selection

\section{Discussion}

\subsection{Feasibility of Pre-portal feature}

This study adopted multi-phase texture features to enhance the precision of MVI classification. Multi-phase texture features like pre-portal feature is designed to eliminate the bias from some noises like gray level or brightness by comparing the slice of different phase in same position. In portal-vein phase of MVI patients, blood with contrast agent would be blocked by MVI in the vein. So, the position of MVI would be darker than its neighborhood. But it is hard to be visible because of the other obvious dark area in the image. In pre-contract phase of MVI phase, MVI position would be slightly darker than its neighborhood, which is hard to be caught by eyes. When getting the differential value between these two phases, the impact factor from the constant area is weaken and the existence of MVI is emphasized.

This method worked well in pre-contract phase and portal vein phase, but did not show the same potential in artery phase. Brightness increment in artery phase rather than other phase could be a responsible reason for it and other possibility still need to be further explored.

\subsection{Validity of NGLDM features}

In the process of feature selection, several NGLDM feature play an important role, which could suggest their close relationship with the presence of MVI.

For Pre feature, functions of Small Dependence Emphasis, Small Dependence Emphasis Low Gray Level Emphasis, Small Dependence Emphasis High Gray Level Emphasis work well, which means the features of small dependence are of vital importance in Pre feature extraction. Small dependence feature 
Predicting Microvascular Invasion of Hepatocellular Carcinoma by texture analysis of ... Q. Yu et al.

reflects the distribution of small dependence. Samples with MVI has a significant higher value in this feature, which means in samples with MVI, textures are much less homogeneous. And without microvascular invasion, vein could have a uniform structure in MR image.

And for Pre-portal feature, Large Dependence Emphasis and Dependence Entropy are the key functions. Samples with MVI has a much lower value in Large Dependence Emphasis than samples without MVI. That is corresponding to the pathological pattern. MR image without MVI would have a more homogeneous portal vein. It also leads to larger and more homogeneous differential between two phases. The same phenomenon also appears in Dependence Entropy.

According to this study, though MVI couldn't be detected in the MR image, its integration could be presented by texture features. Texture features of multi-phase MR image could be a useful approach to predict the presence of MVI in the diagnosis of HCC patients.

\section{Acknowledgment}

This work was supported by the National Key R\&D Project of China grant 2018YFC0910401/2018YFC00910404, NSFC grant 60721003, and Tsinghua-Fuzhou Institute for Data Technology (No. TFIDT2018006).

\section{Reference}

[1] F. Bray, J. Ferlay, I. Soerjomataram, R. L. Siegel, L. A. Torre and A. Jemal, "Global cancer statistics 2018: GLOBOCAN estimates of incidence and mortality worldwide for 36 cancers in 185 countries," a cancer journal for clinicians,, pp. 68(6): 394-424, 2018.

[2] S. Sumie, R. Kuromatsu, K. Okuda, E. Ando, A. Takata, N. Fukushima, ... and M. Sata, "Microvascular invasion in patients with hepatocellular carcinoma and its predictable clinicopathological factors," Annals of surgical oncology, pp. 15(5), 13751382, 2008.

[3] N. F. Esnaola, G. Y. Lauwers, N. Q. Mirza, D. M. Nagorney, D. Doherty, I. Ikai, ... and L. M. Ellis, "Predictors of microvascular invasion in patients with hepatocellular carcinoma who are candidates for orthotopic liver transplantation," Journal of gastrointestinal surgery, pp. 6(2), 224-232, 2002.

[4] S. Roayaie, I. N. Blume, S. N. Thung, M. Guido, M. I. Fiel, S. Hiotis, ... and M. E. Schwartz, "A system of classifying microvascular invasion to predict outcome after resection in patients with hepatocellular carcinoma," Gastroenterology, pp. 137(3), 850-855, 2009.

[5] S. Hwang, Y. Lee, K. Kim, C. Ahn, D. Moon, T. Ha, ... and S. Lee, "The impact of tumor size on long-term survival outcomes after resection of solitary hepatocellular carcinoma: single-institution experience with 2558 patients," Journal of Gastrointestinal Surgery, pp. 19(7), 1281-1290, 2015.

[6] H. Chandarana, E. Robinson, C. H. Hajdu, L. Drozhinin, J. S. Babb and B. Taouli, "Microvascular invasion in hepatocellular carcinoma: is it predictable with pretransplant MRI?," American Journal of Roentgenology, pp. 196(5), 1083-1089, 2011. 
Predicting Microvascular Invasion of Hepatocellular Carcinoma by texture analysis of ... Q. Yu et al.

[7] C. Yang, H. Wang, R. Sheng, Y. Ji, S. Rao and M. Zeng, "Microvascular invasion in hepatocellular carcinoma: is it predictable with a new, preoperative application of diffusion-weighted imaging?," Clinical imaging, pp. 41, 101-105, 2017.

[8] T. Dou, L. Zhang and W. Zhou, "3D deep feature fusion in contrast-enhanced MR for malignancy characterization of hepatocellular carcinoma," in 2018 IEEE 15th International Symposium on Biomedical Imaging , 2018.

[9] S. Men, H. Ju, L. Zhang and W. Zhou, "Prediction Of Microvascular Invasion Of Hepatocellar Carcinoma With Contrast-Enhanced MR Using 3D CNN And LSTM," in 2019 IEEE 16th International Symposium on Biomedical Imaging, 2019.

[10] S. Herlidou-Meme, J. M. Constans, B. Carsin, D. Olivie, P. A. Eliat, L. NadalDesbarats, ... and J. D. De Certaines, "MRI texture analysis on texture test objects, normal brain and intracranial tumors.," Magnetic resonance imaging, pp. 21(9), 989993, 2003.

[11] V. A. Kovalev, F. Kruggel, H. J. Gertz and D. Y. von Cramon, "Three-dimensional texture analysis of MRI brain datasets," IEEE transactions on medical imaging, pp. 20(5), 424-433, 2001.

[12] W. Zhou, L. Zhang, K. Wang, S. Chen, G. Wang, Z. Liu and C. Liang, "Malignancy characterization of hepatocellular carcinomas based on texture analysis of contrast-enhanced MR images," Journal of Magnetic Resonance Imaging, pp. 45(5), 1476-1484., 2017.

[13] J. Zhang, X. Liu, H. Zhang, X. He, Y. Liu, J. Zhou and D. Guo, "Texture Analysis Based on Preoperative Magnetic Resonance Imaging (MRI) and Conventional MRI Features for Predicting the Early Recurrence of Single Hepatocellular Carcinoma after Hepatectomy.," Academic radiology, pp. 26(9), 1164-1173, 2019.

[14] Y. Li, C. Yan, S. Weng, Z. Shi, H. Sun, J. Chen, ... and J. Hong, "Texture analysis of multi-phase MRI images to detect expression of Ki67 in hepatocellular carcinoma," Clinical radiology, pp. 74(10), 813-e19, 2019.

[15] C. Sun and W. G. Wee, "Neighboring gray level dependence matrix for texture classification," Computer Vision, Graphics, and Image Processing, pp. 23(3), 341$352,1983$.

[16] V. Griethuysen, J. J, A. Fedorov, C. Parmar, A. Hosny, N. Aucoin, V. Narayan, ... and $\mathrm{H}$. Aerts, "Computational radiomics system to decode the radiographic phenotype," Cancer research, pp. 77(21), e104-e107, 2017. 